\section{B MIXING and FLAVOR TAGGING at CDF}

\author{
James S. Russ \\ Physics Department, Carnegie Mellon University \\ Pittsburgh, PA 15213 USA \\ for the CDF Collaboration
}

\begin{abstract}
The CDF Collaboration has made a preliminary measurement of $\mathrm{B}_{\mathrm{d}}$ mixing as a first step toward measuring mixing in the $B_{\mathrm{S}}$ system. Flavor tagging using opposite-side jets and muons as well as same-side tagging schemes have been applied. Results agree well with precise results from the B-factories. We use these results to estimate CDF's $B_{S}$ mixing range using the present data set $\left(\sim 250 \mathrm{pb}^{-1}\right)$ and extrapolate to the potential from larger data sets in future running.

Keywords: B Mixing; Flavor Tagging; CDF
\end{abstract}

\section{B Mixing Motivation and Methods}

$\mathrm{CP}$ violation in the Standard Model is controlled by one complex phase appearing both in the s- and b-quark sectors. Mixing in the neutral B meson system offers a means to test the consistency of the unitarity triangle for the b-quark. Measuring the ratio of the mass splitting between the mass eigenstates of the $B_{S}$ and $B_{d}$ particle and antiparticle pairs $\Delta \mathrm{m}_{\mathrm{s}} / \Delta \mathrm{m}_{\mathrm{d}}$, along with lattice gauge calculations of the decay constant $\mathrm{f}$ and the box term $\mathrm{B}$ (factors in [] in Eq.1), gives the length of one side of the unitarity triangle, i.e., $\left|\mathrm{V}_{\mathrm{ts}} / \mathrm{V}_{\mathrm{td}}\right|$, as seen from Eq. 1. This side is already determined by the ensemble of other CP-violating measurements. A disagreement with the B mixing result may be a signal for new physics beyond the standard model. ${ }^{1}$

$$
\frac{\Delta \mathrm{m}_{\mathrm{s}}}{\Delta \mathrm{m}_{\mathrm{d}}}=\frac{\mathrm{m}_{\mathrm{B}_{\mathrm{s}}^{0}}}{\mathrm{~m}_{\mathrm{B}_{\mathrm{d}}^{0}}}\left[\frac{\mathrm{f}_{\mathrm{B}_{\mathrm{s}}^{0}}^{2} \mathrm{~B}_{\mathrm{B}_{\mathrm{s}}^{0}}}{\mathrm{f}_{\mathrm{B}_{\mathrm{d}}^{0}}^{2} \mathrm{~B}_{\mathrm{B}_{\mathrm{d}}^{0}}}\right] \frac{\left|\mathrm{V}_{\mathrm{ts}}\right|^{2}}{\left|\mathrm{~V}_{\mathrm{td}}\right|^{2}} .
$$

\section{Tagging Methods}

In the PDG naming convention, the $B_{d}^{0}$ meson is a $\bar{b} d$ quark pair at its time of production. At later time $t$ the mixing process changes the description of the $\overline{\mathrm{b}}$ quark content to a new linear combination that involves both $\bar{b}$ and $b$-quark states. The mixing rate is controlled by $\Delta \mathrm{m}_{\mathrm{d}}{ }^{1}$.

In this paper I report on three tagging methods applied to semileptonic $\mathrm{B}_{\mathrm{d}}^{0}$ decays: ${ }^{2}$. Experimentally, one observes a $\mathrm{B}$ meson decay at proper time t. The decay mode tags the flavor of the decaying hadron at that time, e.g., $\mathrm{B}_{\mathrm{d}}^{0}$. To measure mixing one needs to know whether it had the same flavor at production. We use 
(a) Opposite side muon tagging $\left(\mu^{+} \Rightarrow\right.$ it was a $\overline{\mathrm{B}}_{\mathrm{d}}^{0}$ at production); (b) Opposite side jet charge tagging (positive jet charge $\Rightarrow$ it was a $\bar{B}_{d}^{0}$ at production); (c) Same side pion tagging $\left(\pi^{-} \Rightarrow\right.$ it was a $\overline{\mathrm{B}}_{\mathrm{d}}^{0}$ at production). We also report a result for fully-reconstructed $\mathrm{B}$ hadronic decays using same-side tagging. The procedures used in this analysis follow directly from CDF Run 1 methods ${ }^{2}$. Details of the tagging method differ for semileptonic and fully-reconstructed decays and will be described later. I will compare the $\Delta \mathrm{m}_{\mathrm{d}}$ and for the tagging efficiency $\epsilon \mathrm{D}^{2}{ }^{2}$ results for the two analyses.

\subsection{Mixing in Semileptonic $\mathrm{B}_{\mathrm{d}}^{0}$ Decays}

At the Tevatron collider B hadrons are produced in multiparticle final states and may well be the daughters of decay chains from higher resonant states. With charge conjugate states implied and using the symbol $\ell$ for either an electron or a muon, we use three decay channels totalling $115 \mathrm{~K}$ events in this analysis: [1] $\mathrm{B}_{\mathrm{d}} \rightarrow \mathrm{D}^{*+} \ell^{-} \mathrm{X} \nu$ $\left(9737 \pm 113\right.$ events); [2] $\mathrm{B}_{\mathrm{d}} \rightarrow \mathrm{D}^{+} \ell^{-} \mathrm{X} \nu$ (35951 \pm 411 events); [3] $\mathrm{B}_{\mathrm{d}} \rightarrow \mathrm{D}^{0} \ell^{-} \mathrm{X} \nu$ $\left(69378 \pm 378\right.$ events). $\mathrm{D}^{+}$mesons are reconstructed using the $\mathrm{K}^{-} \pi^{+} \pi^{+}$final state. $\mathrm{D}^{0}$ mesons are reconstructed using only the $\mathrm{K}^{-} \pi^{+}$final state. The symbol $\mathrm{X}$ refers to possible missing particles in the decay, both charged and neutral. This ambiguity makes both the charge of the parent $\mathrm{B}$ and its momentum uncertain and results in cross-feed between unmixed $\mathrm{B}^{+}$hadrons and (possibly) mixed $\mathrm{B}^{0}$ hadrons as parents of the observed final state $\mathrm{D}^{(*)} \ell$ system. This requires us to understand the b-hadron sample composition, i.e., the mixture of $\mathrm{B}^{+}, \mathrm{B}^{0}, \mathrm{~B}_{\mathrm{S}}^{0}$, and $\Lambda_{b}^{0}$ hadrons produced in $1.96 \mathrm{TeV} \overline{\mathrm{p}}$ collisions. The charged decay pions of excited $\mathrm{B}$ hadrons also complicate the same-side tagging analysis by faking a primary pion produced in close kinematic proximity to the B hadron.

A semileptonic decay event may be tagged by $0,1,2$, or 3 of the tagging methods listed above. If there is only 1 tag available, we determine the dilution parameter $\mathrm{D}$ appropriate for the kinematics of the event and the relevant tagging method. If $\mathrm{P}_{\text {mistag }}$ is the probability of assigning the wrong flavor to a given $\mathrm{B}$ hadron using this tagging method, the dilution $\mathrm{D}=1-2 \mathrm{P}_{\text {mistag. }}$. If several opposite-side tagging methods apply to the current event, we use only one of them chosen from the following hierarchy: ${ }^{2}$

(1) opposite side lepton tag (high purity, low efficiency)

(2) opposite side jet charge tag with evidence of B secondary vertex

(intermediate purity, intermediate efficiency)

(3) opposite side high-p $\mathrm{p}_{\mathrm{T}} \mathrm{b}$ jet tag (low purity, high efficiency)

If we have both an opposite-side tag and a same-side tag for the given event, we combine the dilutions. Because tagging is not perfect ( $\mathrm{P}$ is not zero), the tags may agree or disagree. We allow for each possibility:

$$
D_{\text {agree }}=\frac{D_{\text {opp }}+D_{\text {same }}}{1-D_{\text {opp }} \times D_{\text {same }}} \quad D_{\text {disagree }}=\frac{\left|D_{\text {opp }}-D_{\text {same }}\right|}{1+D_{\text {opp }} \times D_{\text {same }}}
$$


This analysis used a binned $\chi^{2}$ fit for 13 parameters: (a) five dilutions, namely, same-side tagging for $\mathrm{B}^{0}$ and $\mathrm{B}^{+}$, opposite side muon tag, and two opposite-side jet charge tags; (b) three sample composition parameters, Gaussian-constrained to PDG values; (c) two lifetimes, Gaussian-constrained to PDG values for $\tau_{B^{+}}$and $\tau_{B^{0}}$; two efficiency parameters $\left(\mathrm{B}^{+}\right.$and $\left.\mathrm{B}^{0}\right)$; and $\Delta \mathrm{m}_{\mathrm{d}}$. The data are binned into 10 bins of proper time for each tag option (10) and each decay channel (3), giving 300 binned data points to be fit simultaneously. The results give oscillation curves that can be projected back onto each of the 30 tagged decay channels. The fit gives tagging significance quantities $\epsilon \mathrm{D}^{2}$ for the 4 tags. (The two jet-charge taggers have been combined.)

\begin{tabular}{c|c|c|cl}
\hline same-side B $^{+}$ & same-side B $^{0}$ & opposite muon & opposite B-jet & \\
\hline $4.69 \pm 0.38$ & $1.04 \pm 0.24$ & $0.32 \pm 0.05$ & $0.49 \pm 0.07$ & $\%$
\end{tabular}

The result from the semileptonic mixing analysis using 115,000 decays is

$$
\Delta \mathrm{m}_{\mathrm{d}}=0.532 \pm 0.037 \text { (stat) } \pm 0.009(\mathrm{sc}) \pm 0.006(\text { syst }) \mathrm{ps}^{-1}
$$

This result, which quotes the sample composition (sc) error explicitly, agrees well with the B-factory result and has a very small systematic error, due in large part to improved tagging efficiency from extended silicon vertex detector coverage. The tagging efficiency numbers in the collider environment are, unfortunately, much smaller than those for the B-factories. In the BaBar and Belle $\mathrm{CP}$ analyses, for example, the total $\mathrm{B}^{0}$ tagging significance was about $30 \%$ instead of $1.8 \%$ for CDF.

\subsection{Mixing in Fully-Reconstructed Decays}

Semileptonic decays suffer from missing momentum information due to partial reconstruction. This is corrected only on average and smears the proper time information. Fully-reconstructed decays have exact momenta and give precise proper time for each event. The cost is a lower branching fraction for each channel. CDF presents the first hadron collider measurement of $\Delta \mathrm{m}_{\mathrm{d}}$ using fully-reconstructed events in five decay channels: $\mathrm{J} / \psi \mathrm{K}^{* 0} ; \mathrm{D}^{-} \pi^{+} ; \mathrm{D}^{*-} \pi^{+} ; \mathrm{D}^{-} \pi^{+} \pi^{-} \pi^{+} ; \mathrm{D}^{*-} \pi^{+} \pi^{-} \pi^{+}$. In CDF we reconstruct only $\mathrm{D}^{*-} \rightarrow \mathrm{D}^{0} \pi^{-}$; we use both the $\mathrm{K}^{-} \pi^{+}$and $\mathrm{K}^{-} \pi^{+} \pi^{-} \pi^{+}$decay modes of the $\mathrm{D}^{0}$. The analysis is done only with same-side tagging up till now. There are 11,000 fully-reconstructed events in these 5 channels. The B signals are very clean and have good mass resolution.

The fits are done in the same way as the semileptonic fits. We fit all five channels simultaneously, allowing the dilution for each channel to differ. Within statistics, they are the same and are consistent with a fit to a single common dilution. The results of the fit are: $\Delta \mathrm{m}_{\mathrm{d}}=0.526 \pm 0.056$ (stat) \pm 0.006 (syst) $\mathrm{ps}^{-1}$

$$
\epsilon \mathrm{D}^{2}=1.00 \pm 0.35 \text { (stat) } \pm 0.06 \text { (syst) } \%
$$

Both of these results agree well with the semileptonic determination, albeit with larger statistical errors because the sample size is 10 times smaller. Improved tagging methods have already been developed. Preliminary tests indicate that they will 
improve the methods used here by a factor of 1.4. In addition, new tags using time of flight and $\mathrm{dE} / \mathrm{dx}$ particle ID methods for same- and opposite-side kaon tagging are being studied.

\section{Projections for $\mathrm{B}_{\mathrm{S}}$ Mixing at $\mathrm{CDF}$}

The goal of mixing studies is to measure $\Delta \mathrm{m}_{\mathrm{s}} / \Delta \mathrm{m}_{\mathrm{d}}$. Because of the rapid oscillation for $\mathrm{B}_{\mathrm{S}}$ mixing, good proper time resolution is essential. In the $\mathrm{B}_{\mathrm{d}}$ mixing studies we used a run-averaged transverse beam position and ignored the inner silicon detector layer (L00). The average proper time resolution was $67 \mathrm{fs}$. If we find the transverse beam position event-by-event and include L00 (currently only $60 \%$ efficient), the resolution drops to $47 \mathrm{fs}$. Using the actual resolution curves and not just the average, we have run toy Monte Carlo studies of the CDF reach in measuring $\Delta \mathrm{m}_{\mathrm{s}}$ from the present set of 700 fully-reconstructed and 2350 semileptonic $\mathrm{B}_{\mathrm{S}}$ decays, taken in the first $250 \mathrm{pb}^{-1}$ of $\mathrm{CDF}$ data.

We have not yet tried to study $\Delta \mathrm{m}_{\mathrm{s}}$ mixing, so we cannot say with confidence what the tagging efficiency will be for any given tagger. That work is now underway. We have projected our capability on the basis of two scenarios: (a) baseline tagging, projected at $\epsilon \mathrm{D}^{2}=1.6 \%$ and proper time resolution of $67 \mathrm{fs}$; (b) stretched tagging, projecting an $\epsilon \mathrm{D}^{2}=2.6 \%$ and proper time resolution of $47 \mathrm{fs}$. The baseline numbers have already been exceeded for the $B_{d}$ system, but that's not the same as achieving it for $\mathrm{B}_{\mathrm{S}}$ mixing.

Using these two parameter sets, the projected CDF range for a $95 \%$ confidence level upper limit on $\Delta \mathrm{m}_{\mathrm{s}}$ using both fully-reconstructed and semileptonic data ranges from $14 \mathrm{ps}^{-1}$ for the baseline scenario to $24 \mathrm{ps}^{-1}$ for the stretched case. This is a continuum, and we can conceivably exceed the stretched goal using oppositeside kaon tagging. The ICHEP04 best fit to the CKM parameters predicts $\Delta \mathrm{m}_{\mathrm{s}}=$ $21 \mathrm{ps}^{-1}$. From the present data CDF expects to set the best single-experiment limit on $\mathrm{B}_{\mathrm{S}}$ mixing and perhaps to make the first measurement of $\Delta \mathrm{m}_{\mathrm{s}}$.

There will be more data-taking at CDF. What might we do in the future? Because the $\mathrm{B}$ trigger is prescaled at high luminosity, we must project improvements based on the increased $B_{S}$ yield, not just the increased integrated luminosity. If we increase the present data set by a factor of 4 in three more years of running, then our stretched scenario projects a $\mathrm{B}_{\mathrm{S}}$ mixing limit in the range of $\Delta \mathrm{m}_{\mathrm{s}}<33 \mathrm{ps}^{-1}$ at $95 \%$ confidence. This is far out on the high-mass side of the current fits for $\Delta \mathrm{m}_{\mathrm{s}}$. We expect that a $\mathrm{B}_{\mathrm{S}}$ mixing measurement will be made at $\mathrm{CDF}$ in the not-so-distant future if the current Standard Model projections hold true.

\section{References}

1. hep-ph/0201071

2. A. Affolder, et al., Phys. Rev. D61, 072005 (2000) 